\title{
Evaluation of High Performance Concrete by Partial Replacement of Cement with Silica Fume Natural Sand and Manufactured Sand
}

\author{
M.Gomathi ${ }^{1}$ K. Prem Kumar ${ }^{2}$, \\ ${ }^{1,2}$ (Assistant Professor, Civil Engineering Department, Periyar Maniammai University, \\ Thanjavur, India-613403)
}

\begin{abstract}
In this paper the remedial work for scarcity of the building materials are carried out. Instead of conventional materials it is replaced using the manufacturable resources. Those materials are $M$ sand with River sand for the replacement of fine aggregate and also the combination of $M$ sand and silica fume for the replacement of cement. To prove that the strength related properties are much better than the conventional materials. The important strength such as compressive flexural strength are taken into care and proved with the test results. This is to protect the naturally available resources and the ecofriendly materials to save the environment.
\end{abstract}

Keywords: $M$-sand, River sand, Silica fume, High performance concrete

\section{Introduction}

Concrete with the conventional materials are used vastly in our world largely. Mineral admixture are widely used in concrete for various reasons especially for reducing the amount of cement required for making concrete which shows to a reduction in construction cost. The main field of application is as pozzolanic material is for high performance concrete. Silica fume, also known as micro silica, is an amorphous (non-crystalline) polymorph of silicon dioxide. It is an ultrafine powder collected as a by-product of the silicon and ferrosilicon alloy production and consists of spherical with an average particle diameter of $150 \mathrm{~mm}$. This silica fume is used as a partial replacement with cement.

Manufactured Sand is sand produced from crushing of granite stones in required grading to be used for construction purposes as a replacement for river sand. The crushed sand is of cubical shape with grounded edges, washed and graded to as a construction material. The size of manufactured sand (M-Sand) is less than $4.75 \mathrm{~mm}$. It is well graded in the required proportion. It does not contain organic and soluble compound that affects the setting time and properties of cement, thus the required strength of concrete can be maintained. It does not have the presence of impurities such as clay, dust and silt coatings, increase water requirement as in the case of river sand which impair bond between cement paste and aggregate. M-Sand is obtained from specific hard rock (granite) using the state-of-the-art International technology, thus the required property of sand is obtained. M-Sand is cubical in shape and is manufactured using technology like High Carbon steel hit rock and then rock on rock process which is synonymous to that of natural process undergoing in river sand information. Modern and imported machines are used to produce M-Sand to ensure required grading zone for the sand. The main objective of this work is to evaluate the strength of concrete with the replacement of M-sand by river sand, at an addition of silica fume with cement

\section{MATERIALS}

Silica Fume: The American concrete institute (ACI) defines silica fume as a "very fine non crystalline silica produced in electric arc furnaces as a by-product of production of elemental silicon or alloys containing silicon' 'Silica fume is also known as micro silica, condensed silica fume, volatized silica or silica dust. It can exhibit both pozzolanic and cementitious properties. Silica fume has been recognized as a pozzolanic admixture that is effective in enhancing the mechanical properties to a great extent. Addition of silica fume to concrete improves the durability of concrete and also in protecting the embedded steel from corrosion.

Table 1: Properties of Silica fume

\begin{tabular}{|l|c|}
\hline Specific gravity & 2.2 \\
\hline Bulk density & $576 \mathrm{Kg} / \mathrm{m}^{3}$ \\
\hline Size & $0.1 \mathrm{Micron}$ \\
\hline Surface area & $20000 \mathrm{~m}^{2} / \mathrm{Kg}$ \\
\hline $\mathrm{SiO}_{2}$ & $90-96 \%$ \\
\hline $\mathrm{Al}_{2} \mathrm{O}_{3}$ & $0.5-0.8 \%$ \\
\hline
\end{tabular}


M sand: It is well graded in the required proportion. It does not contain organic and soluble compound that affects the setting time and properties of cement, thus the required strength of concrete can be maintained. It does not have the presence of impurities such as clay, dust and silt coatings, increase water requirement as in the case of river sand which impair bond between cement paste and aggregate. Thus, increased quality and durability of concrete. M-Sand is obtained from specific hard rock (granite) using the state-of-the-art International technology, thus the required property of sand is obtained.

Table 2: Properties of $M$ sand and River sand

\begin{tabular}{|l|l|c|c|}
\hline \multirow{2}{*}{ S.No } & \multicolumn{1}{|c|}{ Properties } & \multicolumn{2}{c|}{ Type of sand } \\
\cline { 3 - 4 } & & & M-sand \\
& Textural composition $(\% \mathbf{b y}$ weight) & 28.1 & 6.6 \\
& Coarse sand $(4.75-2.00 \mathrm{~mm})$ & 44.8 & 73.6 \\
& Medium sand $(2.00-0.425 \mathrm{~mm})$ & 27.1 & 19.8 \\
\hline 2. & Fine sand $(0.425-0.075 \mathrm{~mm})$ & 2.63 & 2.67 \\
\hline 3. & Bpecific Gravity & 15.1 & 14.5 \\
\hline 4. & pH Density $\left(\mathbf{k N} / \mathbf{m}^{\mathbf{3}}\right)$ & 10.11 & 8.66 \\
\hline $\mathbf{5 .}$ & Chemical composition of $\mathbf{M}$ sand & $\mathrm{Si}, \mathrm{Al}, \mathrm{Ca}, \mathrm{Mg}$ & \\
& & $\mathrm{Na}, \mathrm{K}, \mathrm{Fe}, \mathrm{etc}$ & \\
\hline
\end{tabular}

Fine Aggregate: Locally available river sand was used as a fine aggregate which passed through $2.36 \mathrm{~mm}$ sieve and retained in $1.18 \mathrm{~mm}$ sieve was used.

Cement used: Ordinary Portland cement of 43 grade was used throughout the work

Water: Potable water was used for mixing and curing purposes

Table 3: Physical test for materials

\begin{tabular}{|c|l|l|l|l|l|}
\hline S.No & Name of the test & Cement & $\begin{array}{l}\text { Coarse } \\
\text { aggregate }\end{array}$ & $\begin{array}{l}\text { Fine } \\
\text { aggregate }\end{array}$ & M Sand \\
\hline 1. & Specific Gravity test & 3.1 & 2.54 & 2.74 & $\mathbf{2 . 6 4}$ \\
\hline 2. & Finess Test & $5 \%$ & & & \\
\hline 3. & Consistency & $31 \%$ & - & & \\
\hline
\end{tabular}

\section{Experimental work and Results}

Mix design: Mix design on recommended guide lines is really a process of making an initial guess at optimum combination of ingredients and final mix proportion is obtained only on the basis of further rail mixes. As mentioned earlier under the project a comparative study is being carried, as such only type on fine aggregate is varied and all other ingredients are kept constant. To arrive at a concrete mix for this study mix design kept constant. To arrive at a concrete mix design for this study M30 concrete was carried as per IS code.

Table 4: Mix Proportion for M30 Grade Concrete (1:1.85:3.2)

\begin{tabular}{|c|c|c|c|}
\hline Water & Cement (kg) & Fine aggregate (kg) & Coarse aggregate (kg) \\
\hline 175 & 389 & 720 & 1257 \\
\hline
\end{tabular}

\section{Mixing, Casting and Curing of Cubes:}

Table 5: Details of Size and Mix Proportion (River sand and M sand)

\begin{tabular}{|c|c|c|c|c|}
\hline $\begin{array}{c}\text { Specimen } \\
\text { Tested }\end{array}$ & $\begin{array}{c}\text { Silica fume in } \\
\text { River sand }\end{array}$ & $\begin{array}{c}\text { Silica fume in M } \\
\text { sand }\end{array}$ & Size (mm) & $\begin{array}{c}\text { No. of } \\
\text { specimens }\end{array}$ \\
\hline \multirow{3}{*}{$\begin{array}{c}\text { Cube } \\
(28 \text { days } \\
\text { compressive } \\
\text { strength) }\end{array}$} & Conventional & conventional & $150 \times 150 \times 150$ & 6 \\
\cline { 2 - 5 } & $5 \%$ replacement & $5 \%$ replacement & $150 \times 150 \times 150$ & 6 \\
\cline { 2 - 5 } & $10 \%$ replacement & $10 \%$ replacement & $150 \times 150 \times 150$ & 6 \\
\cline { 2 - 5 } & $15 \%$ replacement & $15 \%$ replacement & $150 \times 150 \times 150$ & 6 \\
\hline
\end{tabular}

Table 6: Details of PCC Prism Specimen

\begin{tabular}{|c|c|c|c|c|}
\hline $\begin{array}{c}\text { Specimen } \\
\text { Tested }\end{array}$ & $\begin{array}{c}\text { Silica fume in } \\
\text { River sand }\end{array}$ & $\begin{array}{c}\text { Silica fume in } \mathbf{M} \\
\text { sand }\end{array}$ & Size (mm) & $\begin{array}{c}\text { No. of } \\
\text { specimens }\end{array}$ \\
\hline \multirow{3}{*}{$\begin{array}{c}\text { PCC prism } \\
\text { (28 days } \\
\text { compressive } \\
\text { strength) }\end{array}$} & Conventional & conventional & $700 \times 150 \times 150$ & 2 \\
\cline { 2 - 5 } & $5 \%$ replacement & $5 \%$ replacement & $700 \times 150 \times 150$ & 2 \\
\cline { 2 - 5 } & $10 \%$ replacement & $10 \%$ replacement & $700 \times 150 \times 150$ & 2 \\
\cline { 2 - 5 }
\end{tabular}


Table 7: Details of Reinforced Beam Specimen

\begin{tabular}{|c|c|c|c|c|}
\hline $\begin{array}{c}\text { Specimen } \\
\text { Tested }\end{array}$ & $\begin{array}{c}\text { Silica fume in } \\
\text { River sand }\end{array}$ & $\begin{array}{c}\text { Silica fume in } \mathbf{M} \\
\text { sand }\end{array}$ & Size (mm) & $\begin{array}{c}\text { No. of } \\
\text { specimens }\end{array}$ \\
\hline \multirow{3}{*}{$\begin{array}{c}\text { Beam } \\
(28 \text { days } \\
\text { compressive } \\
\text { strength) }\end{array}$} & Conventional & conventional & $1000 \times 150 \times 200$ & 2 \\
\cline { 2 - 5 } & $5 \%$ replacement & $5 \%$ replacement & $1000 \times 150 \times 200$ & 2 \\
\cline { 2 - 5 } & $10 \%$ replacement & $10 \%$ replacement & $1000 \times 150 \times 200$ & 2 \\
\cline { 2 - 5 } & $15 \%$ replacement & $15 \%$ replacement & $1000 \times 150 \times 200$ & 2 \\
\hline
\end{tabular}

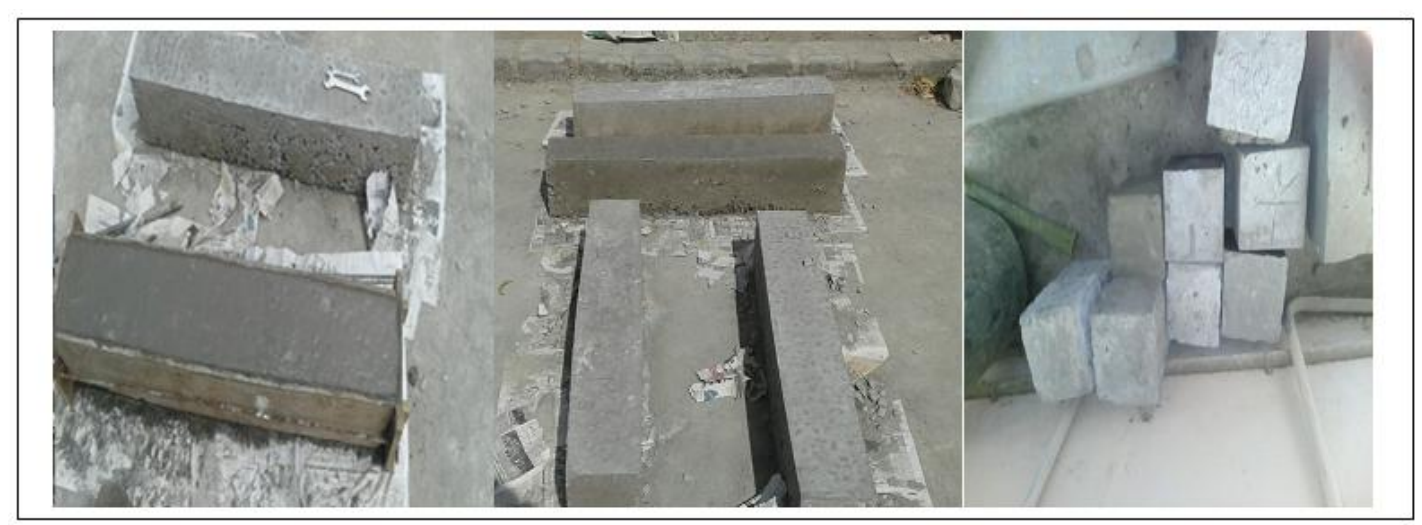

Fig 1: Unmolded specimen

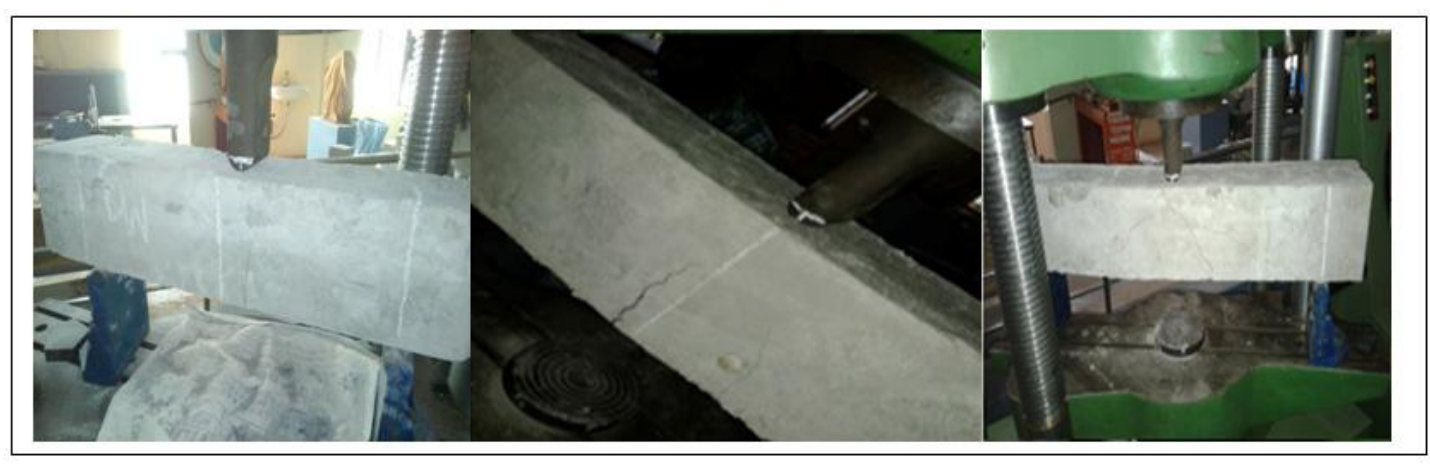

Fig 2: Loading setup for specimen

Table 8: Load Vs Deflection value for River sand and M-sand for Beam

\begin{tabular}{|c|c|c|c|c|c|c|c|c|c|c|}
\hline \multirow[t]{2}{*}{$\begin{array}{c}\text { Load } \\
(\mathbf{k N})\end{array}$} & \multicolumn{2}{|c|}{$\begin{array}{l}\text { Conventional Beam } \\
\text { Deflection }(\mathrm{mm})\end{array}$} & \multicolumn{2}{|c|}{$\begin{array}{l}5 \% \text { silica fume } \\
\text { Deflection }(\mathrm{mm})\end{array}$} & \multicolumn{2}{|c|}{$\begin{array}{l}10 \% \text { silica fume } \\
\text { Deflection } \\
(\mathrm{mm})\end{array}$} & \multicolumn{2}{|c|}{$\begin{array}{l}15 \% \text { silica fume } \\
\text { Deflection } \\
(\mathrm{mm})\end{array}$} & \multicolumn{2}{|c|}{$\begin{array}{l}20 \% \text { silica fume } \\
\text { Deflection } \\
(\mathrm{mm})\end{array}$} \\
\hline & $\begin{array}{l}\text { River } \\
\text { Sand }\end{array}$ & $\begin{array}{c}\text { M- } \\
\text { Sand }\end{array}$ & $\begin{array}{l}\text { River } \\
\text { Sand }\end{array}$ & $\begin{array}{c}\text { M- } \\
\text { Sand }\end{array}$ & $\begin{array}{l}\text { River } \\
\text { Sand }\end{array}$ & $\begin{array}{c}\text { M- } \\
\text { Sand }\end{array}$ & $\begin{array}{l}\text { River } \\
\text { Sand }\end{array}$ & $\begin{array}{c}\text { M- } \\
\text { Sand }\end{array}$ & $\begin{array}{l}\text { River } \\
\text { Sand }\end{array}$ & $\begin{array}{c}\text { M- } \\
\text { Sand }\end{array}$ \\
\hline 5 & 0 & 0 & 0 & 0 & 0 & 0 & 0 & 0 & 0 & 0 \\
\hline 10 & 0.30 & 0.19 & 0.26 & 0.21 & 0 & 0 & 0 & 0 & 0 & 0 \\
\hline 15 & 0.82 & 0.55 & 0.56 & 0.51 & 0.25 & 0 & 0 & 0 & 0 & 0 \\
\hline 20 & 1.04 & 0.78 & 0.85 & 0.70 & 0.64 & 0.55 & 0.10 & 0 & 0 & 0.08 \\
\hline 25 & 1.52 & 1.10 & 1.26 & 1.15 & 1.10 & 1.20 & 0.19 & 0.20 & 0.15 & 0.19 \\
\hline 30 & 1.93 & 1.35 & 1.44 & 1.35 & 1.44 & 1.38 & 0.40 & 0.33 & 0.45 & 0.35 \\
\hline 35 & 2.41 & 1.75 & 1.70 & 1.60 & 1.68 & 1.60 & 0.55 & 0.50 & 0.58 & 0.54 \\
\hline 40 & 3.02 & 2.05 & 2.20 & 2.10 & 2.00 & 1.90 & 0.95 & 0.90 & 0.82 & 0.79 \\
\hline 45 & 3.82 & 2.38 & 2.55 & 2.40 & 2.40 & 2.10 & 1.20 & 1.10 & 1.12 & 1.03 \\
\hline 50 & 4.56 & 3.00 & 2.70 & 2.55 & 2.65 & 2.45 & 1.26 & 1.22 & 1.35 & 1.26 \\
\hline 55 & 5.09 & 3.80 & 2.95 & 2.80 & 2.95 & 2.70 & 1.60 & 1.50 & 1.56 & 1.50 \\
\hline 60 & 5.78 & 4.40 & 3.40 & 3.10 & 3.20 & 3.00 & 2.50 & 2.40 & 1.82 & 1.77 \\
\hline 65 & 6.03 & 5.20 & 3.80 & 3.60 & 3.50 & 3.40 & 3.10 & 3.05 & 1.97 & 1.94 \\
\hline 70 & 6.64 & 5.80 & 4.20 & 3.90 & 4.00 & 3.90 & 3.35 & 3.20 & 2.10 & 2.02 \\
\hline 75 & 7.00 & 6.30 & 4.45 & 4.10 & 4.25 & 4.10 & 3.60 & 3.45 & 2.42 & 2.34 \\
\hline 80 & 7.35 & 6.90 & 4.60 & 4.30 & 4.45 & 4.35 & 3.95 & 3.85 & 2.60 & 2.56 \\
\hline
\end{tabular}




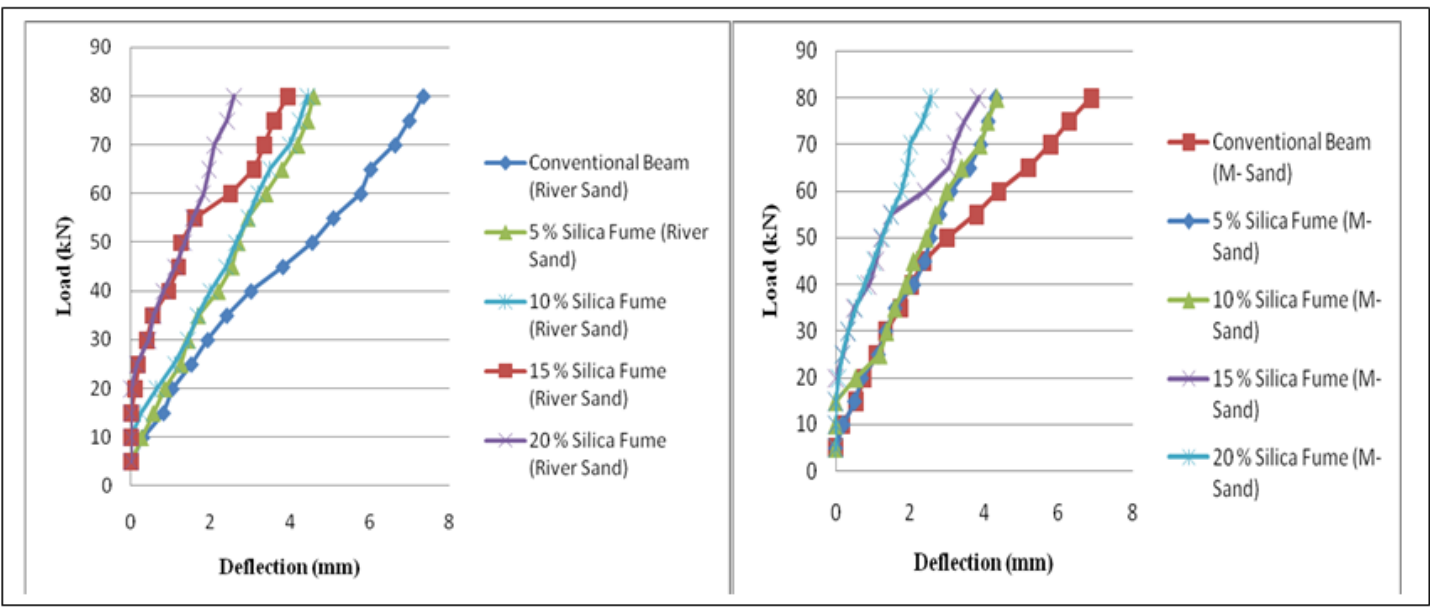

Fig 3: Load Vs Deflection curve for Beam (River sand) Fig 4: Load Vs Deflection curve for Beam (M-sand)

Table 9: Compressive Strength value for Cubes and Flexural Strength value for Prisms

\begin{tabular}{|c|c|c|c|c|c|}
\hline \multirow{2}{*}{ S.No } & Types of specimen & \multicolumn{2}{c|}{ Compressive Strength $\left(\mathbf{N} / \mathbf{m m}^{2}\right)$} & \multicolumn{2}{c|}{ Flexural Strength $\left(\mathbf{N} / \mathbf{m m}^{2}\right)$} \\
\cline { 2 - 6 } & Silica fume & River Sand & M-Sand & River Sand & M-Sand \\
\hline 1 & Conventional & 31.6 & 34.61 & 3.90 & 4.66 \\
\hline 2 & $5 \%$ Replacement & 32.5 & 38.54 & 5.60 & 6.53 \\
\hline 3 & $10 \%$ replacement & 34.0 & 39.75 & 6.84 & 8.01 \\
\hline 4 & $15 \%$ replacement & 36.1 & 41.33 & 10.57 & 11.51 \\
\hline 5 & $20 \%$ replacement & 37.5 & 42.10 & 16.80 & 17.10 \\
\hline
\end{tabular}

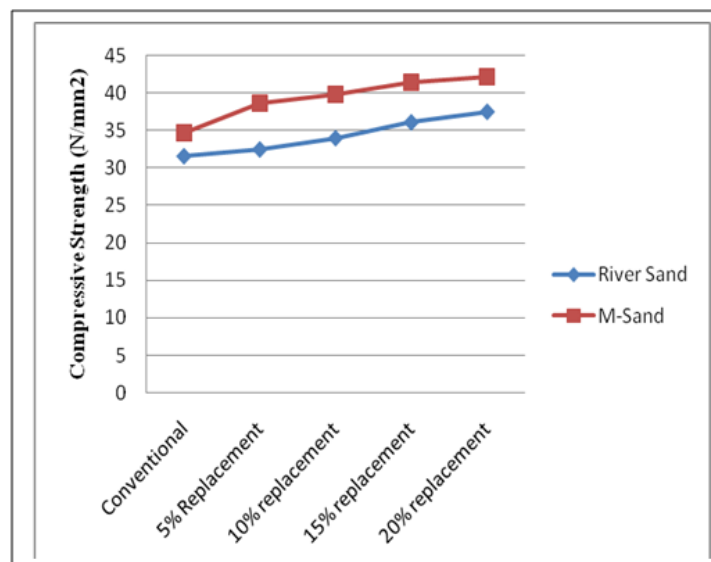

Fig.5 : Compressive Strength Curve for Cubes

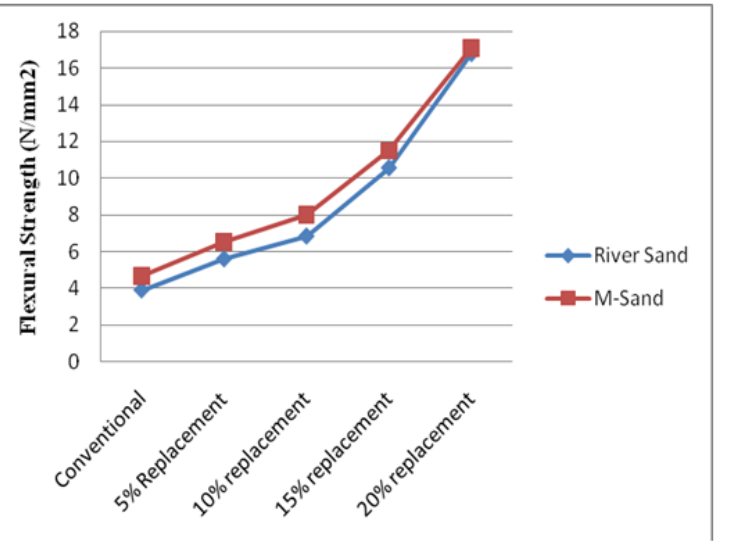

Fig.6 : Flexural Strength Curve for Prisms

\section{Conclusion}

Based on the experimental investigation, the following results have been found. 28 days strength of concrete with M-sand is higher than that of with River sand. Also, due to the superior gradation of M-sand gave good plasticity to mortar providing excellent workability. Silica fume with manufactured sand combination has achieved $5 \%, 10 \%, 15 \%, 20 \%$ higher than the target strength at age of 28 days and other strength parameters such as compressive strength and flexural strength also slightly increased in this combination comparatively. The usage of M-sand for high strength high performance concrete provides stronger and durable concrete structures which will be economical as well as environment friendly by preserving natural resources such as river sand.

\section{References}

[1] Sudarsana Rao.Hunchate, Sashidhar.Chandupall, Vaishali.G.Ghorpode and Venkata Reddy.T.C "Mix Design of High Performance Concrete Using Silica Fume and Superplasticizer" International Journal of Innovative Research in Science, ISSN: 2319-8753,

[2] M. Kaarthik, and K.Subrmanian "Flexural Behavior of Concrete Beams using Recycled Fine Aggregate and Steel Fibres" International Conference on Biological, Civil and Environmental Engineering (BCEE-2014) March 17-18, 2014 Dubai (UAE) 
[3] Arivalagan.S "Flexural Behaviour Of Reinforced Fly Ash Concrete Beams" International Journal of Structural and Civil Engineering ISSN: 2277 7032(Volume 1 Issue 1)

[4] Prof. Vishal S. Ghutke, Prof.PranitaS.Bhandari et.al, "Influence of silica fume on concrete" IOSR Journal of Mechanical and Civil Engineering (IOSR-JMCE) e-ISSN: 2278-1684, P-ISSN: 2320-334X PP 44-47

[5] M.Adams Joe, A.Maria Rajesh, P.Brightson, M.PremAnand - "Experimental Investigation on The Effect Of M-Sand In High Performance Concrete" American Journal of Engineering Research (AJER) e-ISSN : 2320-0847 p-ISSN : 2320-0936 Volume-02, Issue-12, pp-46-51

[6] T. Shanmugapriya, R. N. Uma "Optimization Of Partial Replacement Of M-Sand By Natural Sand In High Performance Concrete With Silica Fume" International Journal of Engineering Sciences \& Emerging Technologies, June 2012. ISSN: 2231 - 6604 Volume 2, Issue 2, pp: 73-80

[7] SudarsanaRao.Hunchate, Sashidhar.Chandupall, Vaishali.G.Ghorpode and VenkataReddy.T.C "Mix Design of High Performance Concrete Using Silica Fume and Superplasticizer" International Journal of Innovative Research in Science, Engineering and Technology, ISSN: 2319-8753 (An ISO 3297: 2007 Certified Organization) Vol. 3, Issue 3, March 2014

[8] MagudeaswaranP ,Eswaramoorthi P "Experimental Investigations of Mechanical properties on Micro silica (Silica Fume) and Fly Ash as Partial Cement Replacement of High Performance Concrete" IOSR Journal of Mechanical and Civil Engineering (IOSRJMCE) ISSN: 2278-1684,p-ISSN: 2320-334X, Volume 6, Issue 4 (May. - Jun. 2013), PP 57-63

[9] Dilip Kumar Singha Roy, Amitava Sil "Effect of Partial Replacement of Cement by Silica Fume on Hardened Concrete" International Journal of Emerging Technology and Advanced Engineering (ISSN 2250-2459, Volume 2, Issue 8, August 2012 ) 472

[10] Indian Standard for Plain And Reinforced Concrete -Code Of Practice ( Fourth Revision ) IS $456: 2000$

M. Gomathi. "Evaluation of High Performance Concrete by Partial Replacement of Cement with Silica Fume Natural Sand and Manufactured Sand ." IOSR Journal of Mechanical and Civil Engineering (IOSR-JMCE) 14.4 (2017): 13-17. 